\title{
Application of Extended IOWA Operator for Making Group Decision on Co-owned Contents in Online Social Networks (OSNs)*
}

\author{
$1^{\text {st }}$ Gulsum Akkuzu \\ School of Computing \\ University of Portsmouth \\ Portsmouth, United Kingdom \\ gulsum.akkuzu@port.ac.uk
}

\author{
$2^{\text {nd }}$ Benjamin Aziz \\ School of Computing \\ University of Portsmouth \\ Portsmouth, United Kingdom \\ benjamin.aziz@port.ac.uk
}

\author{
$3^{\text {rd }}$ Mo Adda \\ School of Computing \\ University of Portsmouth \\ Portsmouth, United Kingdom \\ mo.adda@port.ac.uk
}

\begin{abstract}
Group decision making (GDM) approaches have commonly been used in order to choose the most appropriate choice from an alternative set. It has been applied in environments, where the group of people is involved to make a decision on cases. In online social networks (OSNs), GDM is a need especially on a content of data sharing process where the content of the data has more than one user's information on. In OSNs, social network group decision making (SNGDM) is needed in order to prevent privacy leakage and keep users in OSNs platforms. This is because some OSNs' users might get quit from an OSN platform because of the privacy issues, or they might become unfriend with the user who causes the privacy problem.

Application of having group decision making based OSNs' platforms has a challenge. The challenging part of SNGDM process is to weight users' opinions on their choices on the alternative. One way to weight group members' opinion is to use the trust values. In this paper, we propose an approach where the trust values are used to weight decision-makers' opinions on a co-owned data sharing process. The trust values are used as weight vectors in the Extended Induced Ordered Weighted Average (EIOWA) group decision making technique.

Index Terms-group decision making, online social networks, trust, extended induced ordered weighted average, coowned data sharing, consensus reaching
\end{abstract}

\section{INTRODUCTION}

Group decision making (GDM) is a process in which group of experts make a choice among a set of alternatives. Various approaches have been studied for aggregated opinion of experts, selection of experts' opinion, and for consensus reaching [1]. Experts use their background knowledge and expertises for choosing an alternative from the provided set or making aggregated decision. GDM models consist of two processes, the consensus reaching process (CRP) and the selection process [2]. In a CRP process, decision-makers are let free to change their opinions in order to make their opinions closer to the majority of the group members' decisions or reaching better consensus level. When consensus among decision-makers is reached, a selection process is applied usually by fusing the preferences of individual decision-makers into a collective preference from which the final ordering of the considered alternatives is derived [3].

Increment on the usage of social networks has brought ideas of applying GDM in Online Social Networks (OSNs) environments. OSNs are virtual environments where users can communicate and make connection with others, therefore, social network group decision making (SNGDM) can be applied by using the virtual relationship among users in OSNs' environments [4]. SNGDM is classified into two groups based on the methods that applied in the process. In the first one, decision-makers, who are less knowledgeable and less experienced, might be affected by users with more experience and knowledgeable. The second approach, called dynamic opinion evaluation, in which a decision maker takes the responsibility of forming or evolving other decision-makers' opinions into his own opinion. In our work, we follow the second approach. The process is rounded and decision-makers are allowed to change their opinions till the consensus decision is made [5].

In OSNs, a consensus based group decision making is needed, especially on coowned data sharing process. Since people do not share the contents, which has only their own information on, but also sharing the contents that are related to other people. With these reasons, consensus based group decision making needs to be applied in OSNs' platforms. It can make included users satisfy their opinions are actually considered on the sharing process. In order to apply such process, trust is considered as a key factor in OSNs' environments [6], [7]. In the literature, there are only two works which considers the trust values and opinion dynamic into the consideration to make consensus reached groups decision making [6], [2]. This paper gives a new approach to the consensus reached groups decision making by using trust and reputation values to make the best decision on co-owned data sharing process in OSNs.

The rest of the paper is organised as follows. In Section II, we discuss related work. In Section III, we give the detailed explanation of the proposed work. In Section IV, we give a brief overview of Extended Induced Ordered Average (EIOWA) technique. We then give the application of the proposed work in Section V. Section V presents a case study, which is the implementation of the proposed approach. In Section VI, we conclude the paper and discuss the future work.

\section{RELATED WORK}

Group decision making (GDM) is an important and challenging process, because it includes decision-makers' doubts, problems, and uncertainties [8]. Therefore, finding appropriate ways to help decision-makers is one of the key and critical point in GDM processes. Thirumalai and Senthilkumar [9] propose a fuzzy model to resolve the group decision making problems in business area, the proposed approach uses membership and non-membership attributes to make the group decision. We use fuzzy logic decision making approaches to 
remove uncertainties in group decision making process for OSNs. Fuzzy systems have also been used to solve the group decision making problems in SNs [8].

The consensus process is considered as a repetitive process in which the decision-makers may change their opinions on the alternative set based on the advice given by the moderator (third party) [6]. The advise system includes a feedback mechanism to reduce the inconsistencies knowledge provided by decision-makers [10]. The consensus reaching approaches have been quite productive approaches in SNs. The first social network consensus reaching approach was proposed by Alonso et al. [11], which included a feedback mechanism and delegation mechanism for enhancing the consensus solution.

Li et al. [12] proposed a generalisation of the DeffuantWeisbuch model and studied opinion dynamics in a connected network according to the hard-interaction model and the strategic interaction model. They showed how a required situation guarantees opinion aggregation in the hard interaction model and they also showed how opinion formation processes are affected by the individual incentives behind interactions. Choosing the best parameter for the feedback mechanism has attracted researchers' attentions during recent years [13], [14]. $W u$ et al. proposed a recent work to minimise the changes on decision-makers opinions and reduce the cost of feedback to reach the consensus for group decision making, they also express the trust values with linguistic terms. The usage of the trust values has been one of the resolution for inconsistency of the decision-makers. The importance of the trust values was addressed by [15], [16], it was the first work that applied the trust values to reach satisfied consensus-based decision in SNGDM. Wu et al. presented a new consensus approach that includes a trust based estimation method and an illustrative consensus aggregation model [17]. In order to determine users' weights and to estimate the unknown evaluation values, a relative trust score is used in the proposed work.

CRPs have been improved by researchers day by day with either new models or with applications. An application was proposed by Labella et al [18], the proposed work aimed to resolve the problems in GDM by comparing different GDM models by means of the advantages and disadvantages of the models. We propose a framework for consensus-reached decision in OSNs and we conduct the experimental study of our proposed work with a web application. The proposed work takes the work in [18] as a ground work.

\section{PROPOSED APPROACH}

In this section, we give an overview on the structure of the proposed work. We first explain the detailed steps of a consensus reached group decision making on the co-owned data sharing process in OSNs' platforms (see Figure 1). The owner is the starting point for the process. In the first and the second steps, the owner uploads the content of data and notifies the co-owners with the alternative set, then he waits for group's decision. In the step three, the co-owners select their preferences from the alternative set. Once all the coowners give their preferences on the data sharing process, the system (OSN platform) checks whether taken group decision reached the consensus or not. If not, then the system starts the second round by giving co-owners the same alternative set and a notification "Consensus is not reached: Select the preference alternatives" (see step 4(b) in Figure 1), then the next round is started to reach a consensus based group decision. If consensus is reached in the first round (see Figure 1 step $4(a)$ ), then the system notifies the owner with the consensus reached decision. As a final step owner takes the final decision whether to share the contents with the group's decision or make his own decision, which is different than the group's decision. The system then decreases co-owners' trust in the owner since he does not listen to the group on the co-owned data sharing process. On the other hand, if he listens the group decision, then the system increases co-owners' trusts' values in the owner. We refer readers for trust modelling and its details to [7] [19].

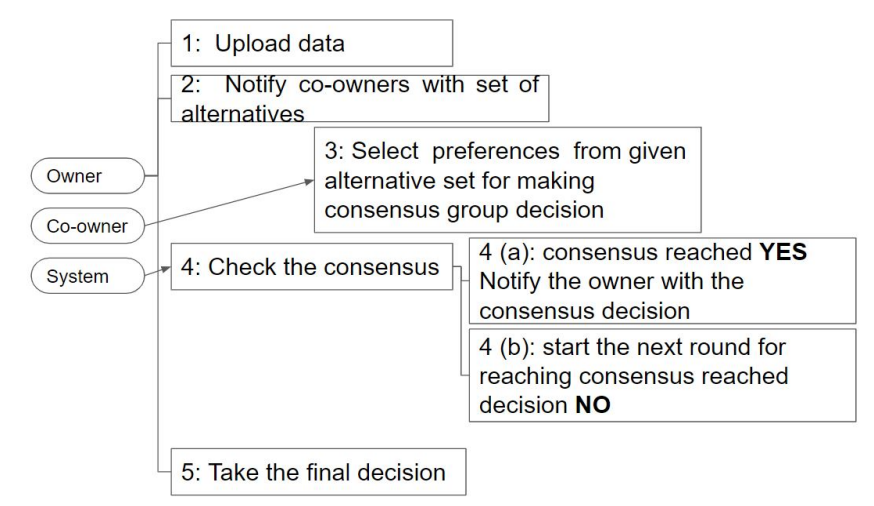

Fig. 1. Structure of the round for consensus reached decision

Figure 2 shows a presentation of the proposed work's trust values between users. Trust value is a number ranges in $[0,1]$. It is a bidirectional variable. In the proposed work, the system assign a starting trust value to users when an edge appears between any two users. Each user has a trust value in other user when they become friends. The trust values are not fixed values, they change based on users' behaviours on co-owned data sharing processes, which refer other users' information. In this work, trust values changes with the final step in Figure 1 ; if the final decision is same with a user's preference, then that user's trust in owner increases. Otherwise, the value of trust in owner goes down.

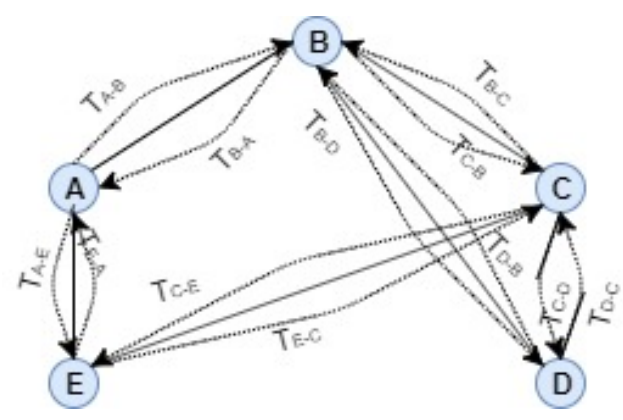

Fig. 2. Representation trust values between user

We give the steps of our method in Figure 3. As it is aforementioned, using the trust values to make a consensusreached decision in OSNs has been an arguable subject. We introduce a new approach to the area, our work uses trust values as weights. We also apply Extended IOWA operator for consensus-reached decision in OSNs. We also develop an OSNs which uses Extended IOWA technique and trust values. Detailed explanation of each step in Figure 3 is explained in the following section (Section V). 

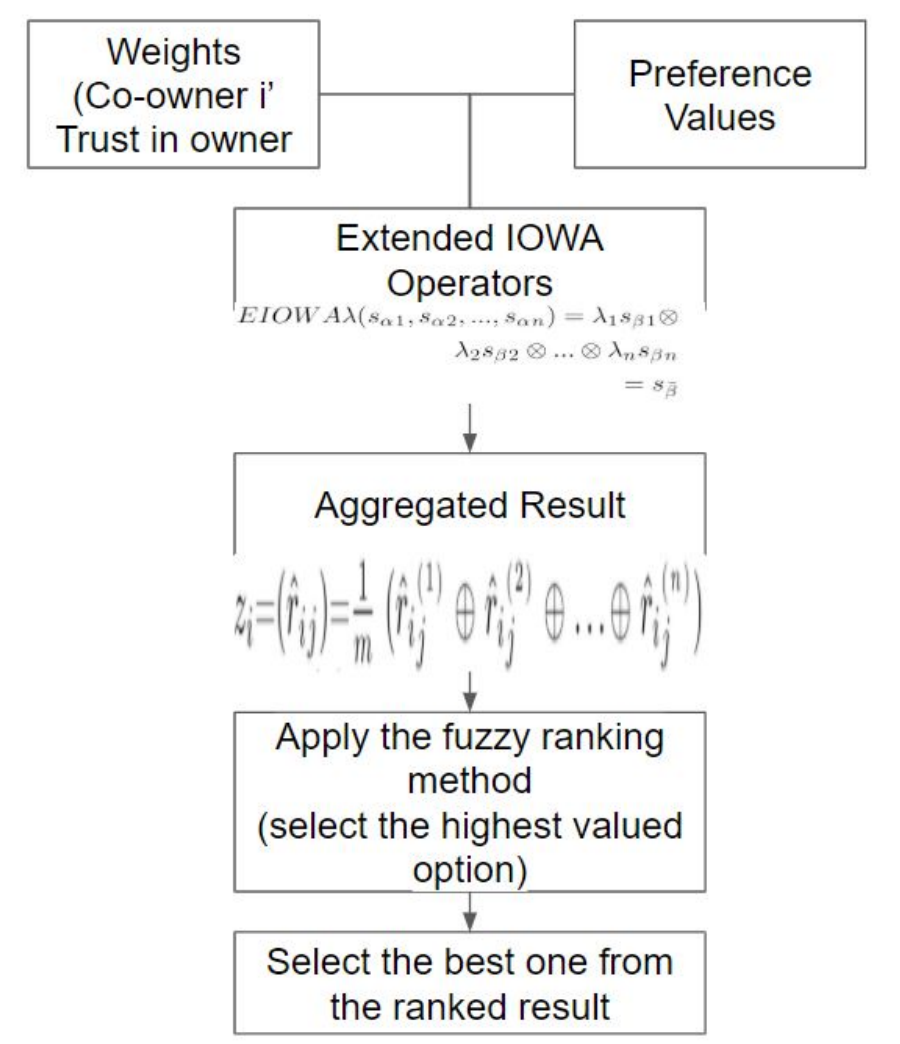

Fig. 3. Extended induced ordered weighted average

\section{EXTENDED INDUCED ORDERED WEIGHTED AVERAGING (EIOWA)}

In this session, we first give an overall explanation of the process of the EIOWA operators' applications in the group decision making with the linguistic preferences. For detailed explanation of the Extended IOWA, we refer readers to [20].

Let $X=\left\{x_{1}, x_{2}, x_{3}, \ldots, x_{n}\right\}$, the alternatives' set, and the group of decision-makers $U=\left\{u_{1}, u_{2}, u_{3}, \ldots, u_{m}\right\}$, and the weighting vector of decision-makers $\lambda=\left\{\lambda_{1}, \lambda_{2}, \lambda_{3}, \ldots, \lambda_{m}\right\}^{T}$, where $\lambda_{l} \geq 0$ and $\sum_{l=1}^{m} \lambda_{m}=1$. Any member of the decision-makers' evaluate the given alternatives with respect to a single linguistic criteria in the set $S=\left\{s_{i} \mid i=-q, \ldots, q\right\}$. Then, put the linguistic preferences in order $R_{k}=\left(r_{i j}^{(k)}\right)_{n x n}$, where $r_{i j}^{(k)} \oplus r_{j}{ }^{(k)}=s_{0}, r_{i}{ }^{(k)}=s_{0}$, $i, j=1,2, \ldots, n$.

The next step is to utilise the EIOWA operator, then get the averaged preference degree of the alternative $i$ th alternative over all the other alternatives. Rank the alternatives and choose the best alternative and finalise the process.

We now demonstrate the steps of the EIOWA technique application on a co-owned data sharing process in OSNs. Decision-makers are co-owners and weighting vectors are the trust values, which exist between the co-owners and the data owner. The data owner is the person, who uploads the content of data to OSNs and responsible for the sharing process. And the content has not only the owner's information but also has other users' information (these users are called co-owners).

- Step (1) Utilise the EIOWA operator (see Equation 1) $\hat{r}_{i j}=E I O W A_{w}\left(r_{i j}^{\left({ }^{(1)}\right.}, r_{i j}^{(2)}, \ldots, r_{i j}^{(l)}\right)$

the parameters values are defined $a=0.5, b=0.5$ and the weights' are valued equally, and thus $w=\frac{1}{n}$ for all $i, j=1,2,3,4, \ldots, \mathrm{n} . \quad i, j=1,2,3,4, \ldots, \mathrm{n}$ are associated to the trust values $(\lambda)$ between the data owner and the DMs.

$$
\begin{array}{r}
E I O W A_{\lambda}\left(s_{\alpha 1}, s_{\alpha 2}, \ldots, s_{\alpha n}\right)=\lambda_{1} s_{\beta 1} \otimes \\
\lambda_{2} s_{\beta 2} \otimes \ldots \otimes \lambda_{n} s_{\beta n} \\
=s_{\bar{\beta}}
\end{array}
$$

where $\bar{\beta}=\sum_{j=1}^{n} \lambda_{j} \beta_{j}, s_{\beta j}$ is the $j$ th largest value of the $s_{\alpha i}$.

- Step (2) Collect all additive linguistic preference relations $R^{(m)}(m=1,2, \ldots, l)$ into an aggregated linguistic preference relation $\hat{R}=\left(\hat{r}_{i j}\right)_{n x n}$

- Step (3) In order to aggregate the preference information $\left(\hat{r}_{i j}\right)$ in the $i t h$ alternative over all the other alternatives utilise the following operator;

$$
z_{i}=\left(\hat{r}_{i j}\right)=\frac{1}{m}\left(\hat{r}_{i j}^{(1)} \oplus \hat{r}_{i j}^{(2)} \oplus \ldots \oplus \hat{r}_{i j}^{(n)}\right)
$$

- Step (4) Rank all the alternative and select the highest valued option from the value of $z_{i}(1,2, \ldots, n)$.

\section{Case Study: Trusty}

In this section, we give an illustrative example of the application of EIOWA group decision making. We applied the technique in Trusty social network. Trusty is an online platform in which users are let to have an account, make friends, share contents (such as photo, text, video), and tag friends. Trust values are assigned to the users when they be friends with other users (as an example of the trust illustration see Figure 2). Each user is also given a reputation value when they have an account in Trusty.

In this work, users account details are hidden for keeping users' information anonymous. We chose six users including owner of the data in order to present the proposed work. Five users give their preferred choices, therefore, it is important to know what are those fives users' trust in owner. We use the trust values from the Trusty's database, each user has trust value in any other user if they are connected to each other. The trust values are held in the system and these values are dynamic.

The sets of alternatives and linguistic labels for each coowned data sharing process in Trusty are as follows;

The set of choices is as follows; $X=$

$$
\left\{\begin{array}{c}
x_{1}=\text { Share with full permission } \\
x_{2}=\text { Share } \text { with restrictions } \\
x_{3}=\text { Share with no permission } \\
x_{4}=\text { Do not share }
\end{array}\right\}
$$

The linguistic labels are given as follows; $S=$

$$
\left\{\begin{array}{cc}
s_{-4} & \text { extremely worried } \\
s_{-3}: & \text { very worried } \\
s_{-2} & \text { worried } \\
s_{-1} & \text { slightly worried } \\
s_{0} & \text { do not mind } \\
s_{1} & \text { slightly agree } \\
s_{2} & \text { agree } \\
s_{3} & \text { fully agree } \\
s_{4} & \text { extremely agree }
\end{array}\right\}
$$

The trust value of the owner in the decision-makers (i.e coowners) are used to weight the decision-makers opinions, see Table I. 
TABLE I

OWNER'S TRUST IN DECISION-MAKERS $\left(\lambda_{o}-D l\right)$

\begin{tabular}{ll}
\hline$D M_{l}$ & The trust value $\lambda_{d l-o}$ \\
\hline $\mathrm{Co}_{1}$ & 0.01 \\
$\mathrm{Co}_{2}$ & 0.02 \\
$\mathrm{Co}_{3}$ & 0.05 \\
$\mathrm{Co}_{4}$ & 0.08 \\
$\mathrm{Co}_{5}$ & 0.02 \\
\hline
\end{tabular}

Table II III, IV, V, and Table VI represent each decisionmakers' preferences from the alternative set.

TABLE II

Linguistic PREFERENCE RELATION $R^{1}$

\begin{tabular}{lllll}
\hline & $x_{1}$ & $x_{2}$ & $x_{3}$ & $x_{4}$ \\
\hline$x_{1}$ & $s_{0}$ & $s_{-3}$ & $s_{-4}$ & $s_{-4}$ \\
$x_{2}$ & $s_{3}$ & $s_{0}$ & $s_{2}$ & $s_{-2}$ \\
$x_{3}$ & $s_{4}$ & $s_{-2}$ & $s_{0}$ & $s_{3}$ \\
$x_{4}$ & $s_{4}$ & $s_{2}$ & $s_{-3}$ & $s_{0}$ \\
\hline
\end{tabular}

TABLE III

Linguistic PREFERENCE RELATION $R^{2}$

\begin{tabular}{lllll}
\hline & $x_{1}$ & $x_{2}$ & $x_{3}$ & $x_{4}$ \\
\hline$x_{1}$ & $s_{0}$ & $s_{1}$ & $s_{-2}$ & $s_{-4}$ \\
$x_{2}$ & $s_{-1}$ & $s_{0}$ & $s_{1}$ & $s_{-2}$ \\
$x_{3}$ & $s_{2}$ & $s_{-1}$ & $s_{0}$ & $s_{4}$ \\
$x_{4}$ & $s_{4}$ & $s_{2}$ & $s_{-4}$ & $s_{0}$ \\
\hline
\end{tabular}

TABLE IV

LINGUISTIC PREFERENCE RELATION $R^{3}$

\begin{tabular}{lllll}
\hline & $x_{1}$ & $x_{2}$ & $x_{3}$ & $x_{4}$ \\
\hline$x_{1}$ & $s_{0}$ & $s_{3}$ & $s_{3}$ & $s_{4}$ \\
$x_{2}$ & $s_{-3}$ & $s_{0}$ & $s_{-3}$ & $s_{-1}$ \\
$x_{3}$ & $s_{-3}$ & $s_{3}$ & $s_{0}$ & $s_{2}$ \\
$x_{4}$ & $s_{-4}$ & $s_{1}$ & $s_{-2}$ & $s_{0}$ \\
\hline
\end{tabular}

TABLE V

Linguistic PREFERENCE RELATION $R^{4}$

\begin{tabular}{lllll}
\hline & $x_{1}$ & $x_{2}$ & $x_{3}$ & $x_{4}$ \\
\hline$x_{1}$ & $s_{0}$ & $s_{2}$ & $s_{-3}$ & $s_{4}$ \\
$x_{2}$ & $s_{-2}$ & $s_{0}$ & $s_{2}$ & $s_{-2}$ \\
$x_{3}$ & $s_{3}$ & $s_{-2}$ & $s_{0}$ & $s_{3}$ \\
$x_{4}$ & $s_{-4}$ & $s_{2}$ & $s_{-3}$ & $s_{0}$ \\
\hline
\end{tabular}

TABLE VI

Linguistic PREFERENCE RELATION $R^{5}$

\begin{tabular}{lllll}
\hline & $x_{1}$ & $x_{2}$ & $x_{3}$ & $x_{4}$ \\
\hline$x_{1}$ & $s_{0}$ & $s_{1}$ & $s_{-2}$ & $s_{-4}$ \\
$x_{2}$ & $s_{-2}$ & $s_{0}$ & $s_{1}$ & $s_{-2}$ \\
$x_{3}$ & $s_{2}$ & $s_{-1}$ & $s_{0}$ & $s_{-4}$ \\
$x_{4}$ & $s_{4}$ & $s_{2}$ & $s_{4}$ & $s_{0}$ \\
\hline
\end{tabular}

Utilising the EIOWA operator

$\hat{r}_{i j}=E I O W A w\left(r_{i j}{ }^{(1)}, r_{i j}{ }^{(2)}, \ldots, r_{i j}{ }^{(m)}\right)$, here we use the $\lambda$

(i.e. trust values). $\lambda$ represents the trust values that exist decision-makers in data owner, $\lambda=T_{o}-{ }_{\text {co }}$. We take the parameter values $a=0.5, b=0.5$ and the values of the weights become $w=0.5,0.5$.

$\lambda_{1}=0.01$ and $\lambda_{2}=0.03, \lambda_{3}=0.05, \lambda_{4}=0.08, \lambda_{5}=0.02$.

Table VII give the clear explanation of the calculation with the preference choices and the trust $(\lambda)$ values.

TABLE VII

CALCULATION FOR THE AGGREGATION MATRIX

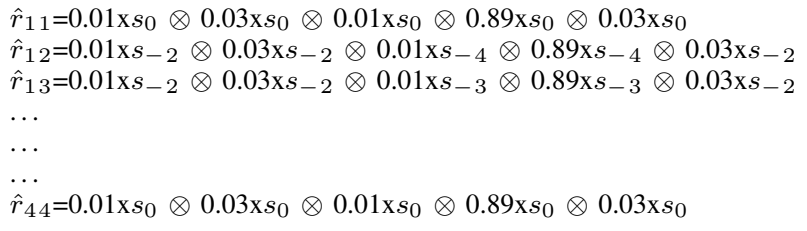

We now use the extended induced ordered weighted average To get best alternative on the data sharing decision-making process in OSNs, we use the EIOWA operator.

TABLE VIII

AGGREGATED PREFERENCE RELATION $R$

\begin{tabular}{lllll}
\hline & $x_{1}$ & $x_{2}$ & $x_{3}$ & $x_{4}$ \\
\hline$x_{1}$ & $s_{0}$ & $s_{-0.14}$ & $s_{-0.11}$ & $s_{-0.08}$ \\
$x_{2}$ & $s_{0.14}$ & $s_{0}$ & $s_{0.08}$ & $s_{-0.05}$ \\
$x_{3}$ & $s_{0.11}$ & $s_{-0.08}$ & $s_{0}$ & $s_{0.01}$ \\
$x_{4}$ & $s_{0.08}$ & $s_{0.05}$ & $s_{-0.1}$ & $s_{0}$ \\
\hline
\end{tabular}

After aggregating the preference relation, we need to sum up the degree of the global preferences.

TABLE IX

THE AVERAGED PREFERENCE DEGREE

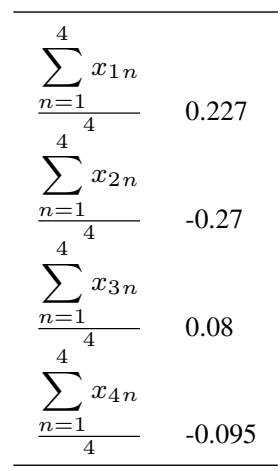

Once the preference degrees are averaged, all the alternatives are ranked accordance with the values of $r_{i}(i=1,2,3,4)$.

Figure 4 shows the ranked alternatives' values changes for the first round. The fluctuation between share with restriction and share with permission is undoubtedly inconsistent.

Table $\mathrm{X}$ shows the highest ranked decision (i.e. best decision is 'share with full permission' with the positive aggregated value, the second ranked decision is 'share with restrictions' with the negative aggregated value, then the third one is 'share with no permission' with positive aggregated value. The distance between share with full permission and share with no permission considerably less than the distance between share with full permission and share with restriction, which is not a consensus-reached decision. This means that the 


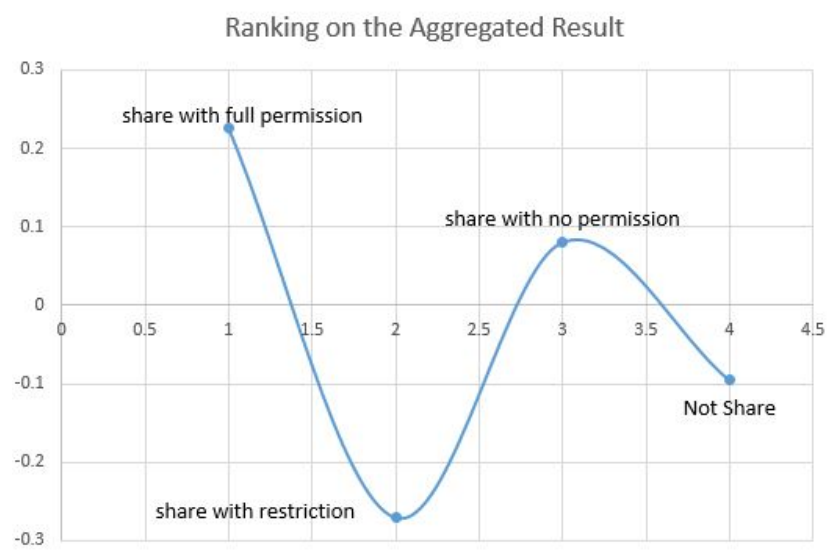

Fig. 4. Ranked aggregated alternative choices

TABLE X

RANKED ALTERNATIVES

$z_{1}>z_{3}>z_{4}>z_{2}$

$x_{1}>x_{3}>x_{4}>x_{2}$

group decision is not reasonable for the coowned data sharing process. Therefore, the second round is required by the system.

As it is above mentioned, Figure 4 and Table $\mathrm{X}$ shows that taken group decision is not a sensible result since there is a big differences between the second alternative and the third alternative in the proposed system. Therefore, the system gives a notification to the group 'Consensus is not reached: Select the preference alternatives' (see Figure 1 in Section III). Then the second round is started to reach consensus based decision. In our system, the second round alternative choices of group members were as follows;

TABLE XI

Round 2: Linguistic PREFERENCE RELATION $R^{1}$

\begin{tabular}{lllll}
\hline & $x_{1}$ & $x_{2}$ & $x_{3}$ & $x_{4}$ \\
\hline$x_{1}$ & $s_{0}$ & $s_{-3}$ & $s_{-3}$ & $s_{-4}$ \\
$x_{2}$ & $s_{3}$ & $s_{0}$ & $s_{-2}$ & $s_{-2}$ \\
$x_{3}$ & $s_{-1}$ & $s_{-2}$ & $s_{0}$ & $s_{3}$ \\
$x_{4}$ & $s_{3}$ & $s_{3}$ & $s_{3}$ & $s_{0}$ \\
\hline
\end{tabular}

TABLE XII

Round 2: Linguistic PREFERENCE RELATION $R^{2}$

\begin{tabular}{lllll}
\hline & $x_{1}$ & $x_{2}$ & $x_{3}$ & $x_{4}$ \\
\hline$x_{1}$ & $s_{0}$ & $s_{-4}$ & $s_{-2}$ & $s_{-3}$ \\
$x_{2}$ & $s_{-1}$ & $s_{0}$ & $s_{-1}$ & $s_{-2}$ \\
$x_{3}$ & $s_{-2}$ & $s_{-2}$ & $s_{0}$ & $s_{-4}$ \\
$x_{4}$ & $s_{4}$ & $s_{4}$ & $s_{4}$ & $s_{0}$ \\
\hline
\end{tabular}

TABLE XIII

Round 2: Linguistic Preference Relation $R^{3}$

\begin{tabular}{lllll}
\hline & $x_{1}$ & $x_{2}$ & $x_{3}$ & $x_{4}$ \\
\hline$x_{1}$ & $s_{0}$ & $s_{-3}$ & $s_{-2}$ & $s_{-4}$ \\
$x_{2}$ & $s_{-3}$ & $s_{0}$ & $s_{-3}$ & $s_{-3}$ \\
$x_{3}$ & $s_{-3}$ & $s_{3}$ & $s_{0}$ & $s_{2}$ \\
$x_{4}$ & $s_{4}$ & $s_{4}$ & $s_{4}$ & $s_{0}$ \\
\hline
\end{tabular}

TABLE XIV

Round 2: Linguistic PREFEREnCE RELATION $R^{4}$

\begin{tabular}{lllll}
\hline & $x_{1}$ & $x_{2}$ & $x_{3}$ & $x_{4}$ \\
\hline$x_{1}$ & $s_{0}$ & $s_{-2}$ & $s_{-3}$ & $s_{-3}$ \\
$x_{2}$ & $s_{-3}$ & $s_{0}$ & $s_{-3}$ & $s_{-2}$ \\
$x_{3}$ & $s_{-3}$ & $s_{3}$ & $s_{0}$ & $s_{2}$ \\
$x_{4}$ & $s_{-4}$ & $s_{-1}$ & $s_{-2}$ & $s_{0}$
\end{tabular}

TABLE XV

Round 2: Linguistic PREFERENCE RELATION $R^{5}$

\begin{tabular}{lllll}
\hline & $x_{1}$ & $x_{2}$ & $x_{3}$ & $x_{4}$ \\
\hline$x_{1}$ & $s_{0}$ & $s_{-1}$ & $s_{-2}$ & $s_{-4}$ \\
$x_{2}$ & $s_{-2}$ & $s_{0}$ & $s_{1}$ & $s_{-2}$ \\
$x_{3}$ & $s_{2}$ & $s_{-1}$ & $s_{0}$ & $s_{-4}$ \\
$x_{4}$ & $s_{-4}$ & $s_{2}$ & $s_{4}$ & $s_{0}$ \\
\hline
\end{tabular}

Table XVI presents the aggregated preference choices for the second round. The system now calculates the averaged values of the aggregated values.

TABLE XVI

Round 2: AGgREGATED PREFERENCE RELATION $R$

\begin{tabular}{lllll}
\hline & $x_{1}$ & $x_{2}$ & $x_{3}$ & $x_{4}$ \\
\hline$x_{1}$ & $s_{0}$ & $s_{-0.44}$ & $s_{-0.45}$ & $s_{-0.63}$ \\
$x_{2}$ & $s_{-0.42}$ & $s_{0}$ & $s_{-0.41}$ & $s_{-0.41}$ \\
$x_{3}$ & $s_{-0.4}$ & $s_{0.31}$ & $s_{0}$ & $s_{0.07}$ \\
$x_{4}$ & $s_{-0.09}$ & $s_{0.27}$ & $s_{0.23}$ & $s_{0}$ \\
\hline
\end{tabular}

On the following table, we give the averaged values of the aggregated preference choice shown on Table XVI.

TABLE XVII

ROUND 2: THE AVERAGED PREFERENCE DEGREE

\begin{tabular}{ll}
\hline$\frac{\sum_{n=1}^{4} x_{1 n}}{4}$ & -0.37 \\
$\frac{\sum_{n=1}^{4} x_{2 n}}{4}$ & -0.31 \\
$\frac{\sum_{n=1}^{4} x_{3 n}}{\sum_{n=1}^{4} x_{4 n}}$ & -0.00 \\
\hline
\end{tabular}

Table XVIII gives the ranked alternatives. As it can be seen on the table and Figure 5 the best decision from the group is 'Not Share' choice.

TABLE XVIII

ROUND 2: RANKED ALTERNATIVES

$$
\begin{aligned}
& z_{4}>z_{3}>z_{2}>z_{1} \\
& x_{4}>x_{3}>x_{2}>x_{1}
\end{aligned}
$$

Figure 5 presents the ranked alternatives' values changes. The change between share with restriction and share with no permission is quite interesting when the distance is compared between share with full permission- share with restriction 
and share with no permission-Not share. We can make an interpretation on the case; co-owners certainly do not want the content be shared with any permission. This is most probably related to the high sensitivity of the data.

\section{Ranking on the Aggregated Result}

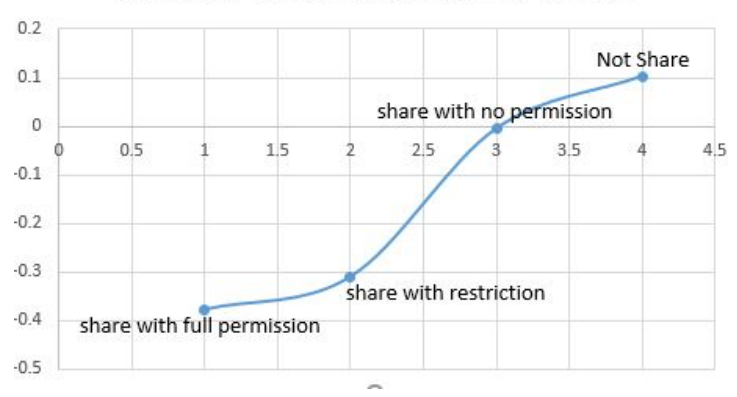

Fig. 5. Round2: Ranked aggregated alternative choices

\section{CONCLUSION}

This work introduces a group decision making on coowned data sharing processes for OSNs' platforms with the usage of Extended Induced Ordered Weighted Average (EIOWA) technique. One of the GDM's main advantages is that it provides an environment where the group of people can express their opinions on a given alternative set in order to make an appropriate decision for the group. The application of the GDM in social network environments is possible with the usage of connection among OSNs' users.

Our work brings a view for co-owned data sharing process in OSNs. The proposed work demonstrates the way to use the EIOWA technique to make a consensus-reached group decision in OSNs' platforms in order to protect privacy leakages, keeping users OSNs' friendship and their online accounts. The usage of the trust values, which exist between OSNs' platforms' users, has been a problem in the literature. Our work demonstrates a solution for the problem and it also covers the gap. We applied the proposed work in an OSN platform (Trusty). This work demonstrates not only the possibility of trust values as weight vectors but also shows that applying the GDM processes in OSNs' platform might protect the privacy protection of OSNs' users. In future research, we will consider usage of other GDM operators in terms of the use of different measures.

\section{REFERENCES}

[1] N. Capuano, F. Chiclana, H. Fujita, E. Herrera-Viedma, and V. Loia, "Fuzzy group decision making with incomplete information guided by social influence," IEEE Transactions on Fuzzy Systems, vol. 26, no. 3, pp. 1704-1718, 2017.

[2] Y. Dong, Q. Zha, H. Zhang, G. Kou, H. Fujita, F. Chiclana, and E. Herrera-Viedma, "Consensus reaching in social network group decision making: Research paradigms and challenges," Knowledge-Based Systems, vol. 162, pp. 3-13, 2018.

[3] E. Herrera-Viedma, F. J. Cabrerizo, J. Kacprzyk, and W. Pedrycz, "A review of soft consensus models in a fuzzy environment," Information Fusion, vol. 17, pp. 4-13, 2014.

[4] J. Wu, F. Chiclana, H. Fujita, and E. Herrera-Viedma, "A visual interaction consensus model for social network group decision making with trust propagation," Knowledge-Based Systems, vol. 122, pp. 39-50, 2017.

[5] W. D. Cook and M. Kress, "Ordinal ranking with intensity of preference," Management science, vol. 31, no. 1, pp. 26-32, 1985.

[6] E. Herrera-Viedma, F. J. Cabrerizo, F. Chiclana, J. Wu, M. J. Cobo, and S. Konstantin, "Consensus in group decision making and social networks," 2017.
[7] G. Akkuzu, B. Aziz, and M. Adda, "Fuzzy logic decision based collaborative privacy management framework for online social networks," in $3 \mathrm{rd}$ International Workshop on FORmal methods for Security Engineering: ForSE 2019. SciTePress, 2019.

[8] Q. Liang, X. Liao, and J. Liu, "A social ties-based approach for group decision-making problems with incomplete additive preference relations," Knowledge-Based Systems, vol. 119, pp. 68-86, 2017.

[9] C. Thirumalai and M. Senthilkumar, "An assessment framework of intuitionistic fuzzy network for c2b decision making," in 2017 4th International Conference on Electronics and Communication Systems (ICECS). IEEE, 2017, pp. 164-167.

[10] Y. Dong, X. Chen, and F. Herrera, "Minimizing adjusted simple terms in the consensus reaching process with hesitant linguistic assessments in group decision making," Information Sciences, vol. 297, pp. 95-117, 2015.

[11] S. Alonso, I. J. Pérez, F. J. Cabrerizo, and E. Herrera-Viedma, "A linguistic consensus model for web 2.0 communities," Applied Soft Computing, vol. 13, no. 1, pp. 149-157, 2013.

[12] L. Li, A. Scaglione, A. Swami, and Q. Zhao, "Consensus, polarization and clustering of opinions in social networks," IEEE Journal on Selected Areas in Communications, vol. 31, no. 6, pp. 1072-1083, 2013.

[13] J. Wu, L. Dai, F. Chiclana, H. Fujita, and E. Herrera-Viedma, "A minimum adjustment cost feedback mechanism based consensus model for group decision making under social network with distributed linguistic trust," Information Fusion, vol. 41, pp. 232-242, 2018.

[14] J. Wu, Y. Liu, and C. Liang, "A consensus-and harmony-based feedback mechanism for multiple attribute group decision making with correlated intuitionistic fuzzy sets," International Transactions in Operational Research, vol. 22, no. 6, pp. 1033-1054, 2015.

[15] E. Herrera-Viedma, S. Alonso, F. Chiclana, and F. Herrera, "A consensus model for group decision making with incomplete fuzzy preference relations," IEEE Transactions on fuzzy Systems, vol. 15, no. 5, pp. 863$877,2007$.

[16] R. Urena, G. Kou, Y. Dong, F. Chiclana, and E. Herrera-Viedma, "A review on trust propagation and opinion dynamics in social networks and group decision making frameworks," Information Sciences, vol. 478, pp. 461-475, 2019.

[17] J. Wu, F. Chiclana, and E. Herrera-Viedma, "Trust based consensus model for social network in an incomplete linguistic information context," Applied Soft Computing, vol. 35, pp. 827-839, 2015.

[18] Á. Labella, F. J. Estrella, and L. Martínez, "Afryca 2.0: an improved analysis framework for consensus reaching processes," Progress in Artificial Intelligence, vol. 6, no. 2, pp. 181-194, 2017.

[19] G. Akkuzu, B. Aziz, and M. Adda, "Advantages of having users' trust and reputation values on data sharing process in online social networks," in The Sixth IEEE International Conference on Social Networks Analysis, Management and Security. IEEE, 2019.

[20] G. Qian and Z.-S. Xu, "Extended iowa operator and its application to group decision making with linguistic preference information," in 2006 International Conference on Machine Learning and Cybernetics. IEEE, 2006, pp. 1662-1666. 